\title{
Solution-processed thin films of a charge transfer complex for ambipolar field-effect transistors
}

\author{
Tommaso Salzillo, ${ }^{\text {ab }}$ Antonio Campos ${ }^{a}$ and Marta Mas-Torrent ${ }^{\mathrm{a}, *}$
}

Charge transfer (CT) complexes are raising a great interest as potential candidate ambipolar semiconductors. However, their application in organic fieldeffect transistors (OFETs) has been limited to ideal single crystals or to films prepared by the co-evaporated of their components, hindering their potential for real applications. Here, we report the preparation of thin films of the CT complex DBTTF-TCNQ by a simple solution shearing technique. This was achieved by blending the active materials with polystyrene to promote film processability, reproducibility and stability. Infrared and Raman Spectrosocopy were used, respectively, for charge transfer degree estimation and structural identification of the films. The resulting OFET devices, fabricated and measured completely in environmental conditions, exhibited an ambipolar field-effect. Thus, this work opens new perspectives for the application of CT salts in electronic devices

\footnotetext{
a. Department of Molecular Nanoscience and Organic Materials, Institut de Ciència de Materials de Barcelona (ICMAB-CSIC) and (CIBER-BBN), Campus de la UAB, 08193 Bellaterra, Spain.

${ }^{b .}$ Universidad Autònoma de Barcelona, Bellaterra (Cerdanyola del Vallès), 08193, Spain.

*Corresponding author: mmas@icmab.es
}

\section{Introduction}

Organic semiconducting small molecules have vastly been employed as active materials in organic field-effect transistors (OFETs) in the last decades for their potential in a wide variety of applications requiring low cost, compatibility with flexible substrates and a favourable interaction with biological media. ${ }^{1-3}$ Although the highest field-effect mobilities are found in ideal single crystal devices, ${ }^{4,5}$ in order to move towards applications many efforts have been devoted to the preparation of thin films using low-cost printing techniques. ${ }^{6,7}$ Nowadays, thin films of semiconductors fabricated employing high throughput processing techniques and achieving excellent device performances have been reported. ${ }^{8,9}$ However, most of these devices are based on only one molecular component and, thus, they typically reveal unipolar character, either $\mathrm{p}$ type or n-type. Indeed, the number of suitable materials able to conduct both holes and electrons is still limited. ${ }^{10,11}$ Some approaches have been focused on fabricating ambipolar devices by preparing multilayer or bulk heterostructures of two different organic semiconductors ( $\mathrm{n}$ - and $\mathrm{p}-)^{12-14}$ Nevertheless, the control of such heterojunctions, especially when employing solution based deposition techniques, is not straightforward.

Alternatively, charge transfer (CT) complexes are raising an increased interest as potential candidate ambipolar semiconductors. Organic CT complexes are materials made up by electron donor (D) and electron acceptor (A) molecules arranged in different configurations such as mixed, where the two chemical moieties alternate in the column in a fashion DADADA, or segregated stacks, where the two species form two columns DDD and AAA. Depending on the different D/A ratios, the polymorphic structure in the solid state and charge transfer degree, it is possible to have a variety of materials with peculiar electronic properties. ${ }^{15-17}$ It has been found that single crystals of neutral or quasi neutral CT complexes can exhibit unique ambipolar OFET characteristics, thanks to the strong donor-acceptor interactions and concomitant low band- gap energy. ${ }^{18-23}$ Despite the encouraging reported results, ${ }^{24-26}$ these studies have been mainly restricted to prototypical single crystals. The preparation of more applicable CT thin films has only been attempted by co-evaporation of their components, ${ }^{27}$ since the low solubility of the donor-acceptor mixtures ${ }^{28-30}$ importantly hampers the solution processability of these materials. In order to make CT complexes promising for applications it is thus imperative to find methods to print them on large areas and employing techniques compatible with up-scaling.

In particular, the CT complex dibenzotetrathiafulvalene7,7,8,8-tetracyanoquinodimethane (DBTTF-TCNQ) has raised a lot of interest as ambipolar semiconductor candidate. ${ }^{18,31-34}$ Single crystals of this material have revealed hole and electron mobilities of the order $10^{-2}-10^{-1} \mathrm{~cm}^{2} \mathrm{~V}^{-1} \mathrm{~s}^{-1} .{ }^{18,31}$ Further, theoretical studies have also predicted extremely high hole and electron mobilities in such crystals. ${ }^{23}$ Here, we report the fabrication of solution-processed OFETs based on thin films of the DBTTF-TCNQ binary system by means of blending the components with an insulating polymer matrix (Figure 1) and depositing the resulting ink with a solution shearing technique compatible with roll-to-roll manufacturing processes. The role of the binding polymer is to promote film processability and crystallinity as well as enhance the device stability. The prepared films, fabricated and measured completely in environmental conditions which is highly desirable for low-cost<smiles>N#CC(C#N)=c1ccc(=C(C#N)C#N)cc1</smiles>
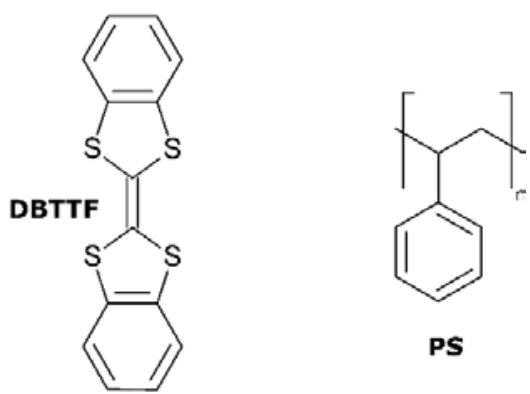

electronics, clearly exhibit ambipolar transport, elucidating Figure 1. Chemical structures of the acceptor TCNQ, the donor DBTTF and the polymer blending agent PS. 
that CT complexes can be promising active electronic materials not only for performing fundamental studies but also for their implementation into real applications.

\section{Experimental}

\section{Materials and methods}

DBTTF (Sigma Aldrich, purity 97\%), TCNQ (Sigma Aldrich, purity $98 \%)$ were sublimed under vacuum before to be used. The polystyrene with $\mathrm{Mw}=280.000 \mathrm{~g} / \mathrm{mol}$ (PS280K, Sigma Aldrich) was used without further purification. All the solvents (from Aldrich) were of spectroscopic grade. The single crystals of the CT complex were obtained by dissolving equimolar amounts of DBTTF and TCNQ in different solvent and solvent mixtures, sonicated and then kept at $353 \mathrm{~K}$ for one hour to ensure the complete dissolution of the parent compounds. Dark green solutions were obtained which were left to cool down to room temperature in sealed vials. Black needle and platelet like crystals (Figure S1) were obtained from the different solvent mixture and checked by single crystal X-ray diffraction.

\section{Transistor fabrication and characterization}

OFETs were fabricated in a bottom-gate/bottom-contact configuration with interdigitated pattern of $\mathrm{Cr} / \mathrm{Au}$ source and drain electrodes keeping constant the Width/Length $(W / L=100)$ ratio and varying the length of the channels from 25 to $100 \mu \mathrm{m}$. The interdigitated patterns were fabricated on highly $\mathrm{n}$-doped $\mathrm{Si}$ wafers with $200 \mathrm{~nm}$ of thermally grown $\mathrm{SiO}_{2}$ purchased from SiMat by photolithography using a MicroWriter ML3 from Durham Magneto Optics Ltd. $\mathrm{Cr}(5 \mathrm{~nm})$ and $\mathrm{Au}(40 \mathrm{~nm})$ layer were deposited by thermal evaporation and, subsequently, the residual photoresist was removed by cleaning the substrates in ultrasonic bath with acetone and isopropanol and then dried with nitrogen. The CT complex polymer blended was deposited on the substrates by Barassisted meniscus Shearing (BAMS), ${ }^{35,36}$ using a home-designed equipment. As described in previous works, this technique uses a smooth cylindrical metallic bar placed $300 \mu \mathrm{m}$ above the heated substrate and the OSC solution is placed between the bar and the substrate to form a confined meniscus. Under the controlled uniform movement of the bar the solution meniscus is displaced and leaves behind an organic thin film formed through evaporation-induced self-assembly. For the preparation of the CT complex thin film, a solution of $20 \mathrm{~g} / \mathrm{l}$ and weight ratio 1:2 DBTTF-TCNQ:PS280K (with equimolar ratio between Donor and Acceptor molecules) in cholorobenzene:benzonitrile (50/50) was deposited at $1 \mathrm{~mm} / \mathrm{s}$ and at 150 oC. It should be noticed in these conditions and at this temperature all the molecular components and the CT complex do not precipitate. For the deposition of the pure TCNQ a $8 \mathrm{~g} / \mathrm{l}$ solution in chlorobenzene was deposited at 10 $\mathrm{mm} / \mathrm{s}$ and $105 \% \mathrm{C}$, while for the TCNQ:PS280K blend a $2 \% \mathrm{wt}$. of an anisole solution with a 3:1 weight ratio was deposited at 10 $\mathrm{mm} / \mathrm{s}$ and 105 oc.

The field-effect transistors were characterized in air and in dark conditions with an Agilent B1500A semiconductor parameter analyser connected to the sample with a Karl SüsS probe station. The highly-doped Silicon was used as gate and the field-effect mobilities $(\mu)$ were calculated from the saturation regions using the following equation:

$$
I_{S D}=\frac{W C_{i} \mu}{2 L}\left(V_{G S}-V_{t h}\right)^{2}
$$

where $\mathrm{W}$ and $\mathrm{L}$ are the channel width and length, respectively, $C_{i}$ is the capacitance of the dielectric layer and $V_{t h}$ is the threshold voltage.

\section{Spectroscopic measurements}

Raman spectra were recorded with a Horiba XploRA PLUS spectrometer equipped with a suitable edge filter and coupled to an Olympus BX51 confocal microscope with 100x and 10x objectives which allow for a lateral resolution lower than $1 \mu \mathrm{m}$ with the higher magnification. This experimental configuration allows gathering information on the polymorphic composition in crystal domains of micrometric dimensions. The excitation wavelength used was the $532 \mathrm{~nm}$ line from a Diode-Pumped Solid State (DPSS), with a nominal power of $35 \mathrm{~mW}$. The power was reduced by neutral density filters to avoid sample damage. The Horiba LabSpec 6 software was used to operate the spectrometer.

Infrared Reflection-Absorption Spectroscopy (IRRAS) spectra were recorded by using a Bruker Vertex 70 spectrometer with PM50 module equipped with a liquid-nitrogen-cooled mercury-cadmium-telluride (MCT) photodetector and a permanently aligned Rock Solid interferometer. To reduce absorption due to water and carbon dioxide, the spectrometer was purged by fluxing nitrogen gas. The Bruker spectroscopy software OPUS, version 4.2, was used to operate the spectrometer. Reflection measurements were performed with an aperture of $6 \mathrm{~mm}$ in diameter on the primary side and on aperture of the secondary side at a resolution of $2 \mathrm{~cm}^{-1}$. The incidence angle was set to the Brewster angle of the $\mathrm{Si} / \mathrm{SiO}_{2}$ substrate at 75 o to get the best signal-to-noise ratio.

\section{Structural and morphological characterizations}

The crystal structure of the dark black needle-like specimen of DBTTF-TCNQ was solved by single crystal X-ray diffraction (XRD). The X-ray intensity data were measured on a Bruker D8 QUEST ECO system equipped with a doubly curved silicon crystal Bruker Triumph monochromator and a Mo Ka sealed Xray tube $(\lambda=0.71073 \AA)$. The frames were integrated with the Bruker SAINT software package using a wide-frame algorithm. Data were corrected for absorption effects using the MultiScan method (SADABS). Crystallographic data for the reported structure has been deposited with the Cambridge Crystallographic Data Centre (CCDC) and the cell parameters are reported in the following Table 1 . Reference numbers CCDC 1897001.

Table 1 Cell parameters for DBTTF/TCNQ crystal obtained from solution.

$\begin{array}{ll}\text { DBTTF-TCNQ } & 1: 1 \\ \text { Empirical formula } & \mathrm{C}_{26} \mathrm{H}_{12} \mathrm{~N}_{4} \mathrm{~S}_{4} \\ \text { Formula weight } & 508.64 \mathrm{~g} / \mathrm{mol} \\ \text { Temperature } & 303(2) \mathrm{K} \\ \text { Wavelength } & 0.71073 \AA \\ \text { Crystal system } & \text { Triclinic }\end{array}$




$\begin{array}{lll}\text { Space group } & \mathrm{P}-1 & \\ \text { Unit cell } & a=7.7333(8) \AA & \alpha=72.079(3)^{\circ} \\ \text { dimensions } & b=8.2925(9) \AA & \beta=70.193(3)^{\circ} \\ & c=10.3947(11) \AA & \gamma=70.053(3)^{\circ} \\ \text { Volume } & 575.46(11) \AA^{3} & \\ Z & 1 & \end{array}$

Film XRD diffractograms were collected in the $2 \theta$ range between $5^{\circ}$ and $40^{\circ}$. This was done on a Siemens, model D5000 diffractometer with Bragg-Brentano geometry equipped with a Scintillator detector, in the 20 range $2.5-40^{\circ}$, using CuK $\alpha$ radiation (step size, $0.02^{\circ}$; time/step, $2 \mathrm{~s} ; 45 \mathrm{kV} \times 35 \mathrm{~mA}$ ).

Optical microscope images were taken with a B-600 MET from OPTIKA in crossed polarizers configuration. Atomic force microscope (AFM) images were obtained working with a 5100 SPM system from Agilent technologies in tapping mode and the images were analyzed using Gwyddion 2.47 software.

\section{Results and discussion}

The use of solution shearing techniques for the deposition of organic semiconductors (OSCs) is currently raising great interest for developing low cost and highly reproducible devices. ${ }^{7,29,37,38}$ Particularly, our group has reported the use of the bar-assisted meniscus shearing technique for preparing high performing OFETs based on $\mathrm{p}$ - and $\mathrm{n}$-type small molecule OSCs. ${ }^{35,36,39}$ This technique consists in forming a meniscus of an OSC semiconductor solution in between a heated substrate and a bar. Subsequently, the bar is dragged at a constant speed leaving behind a crystalline and homogenous film. Additionally, it has been shown that blending the OSC with the insulating polymer polystyrene (PS) provides additional advantages such as promoting the self-organization of the semiconductor molecule by vertical phase separation, improving the films homogeneity and enhancing the device stability by self-encapsulating the OSC and by passivating the hydroxyl groups of the dielectric layer that act as electron trapping centres hindering the electron transport. ${ }^{39}$

Previously, we reported single crystal-like performance in $p$ type OFETS based on thin films of DBTTF blended with PS and deposited by BAMS (105다. $10 \mathrm{~mm} / \mathrm{s})$ using chlorobenzene ink solutions. ${ }^{35,40}$ Importantly to note is the fact that the use of PS permitted to fabricate and measure the OFETs in environmental conditions, since devices based on only DBTTF were not stable in air. Thus, prior to the preparation of CT films we proceed in fabricating OFETs based on the acceptor TCNQ component. Single crystals of TCNQ have been reported to display a n-type mobilities of up to $1 \mathrm{~cm}^{2} \mathrm{~V}^{-1} \mathrm{~s}^{-1,4,41}$ although, to our knowledge, there are no precedents of its deposition using solution shearing techniques. Chlorobenzene solutions of bare TCNQ $(8 \mathrm{~g} / \mathrm{l})$ were deposited by BAMS $(105$ o $C, 10 \mathrm{~mm} / \mathrm{s})$ on $\mathrm{Si} / \mathrm{SiO}_{2}$ substrates giving rise to highly crystalline and homogenous thin films with long crystallites oriented along the deposition direction, as shown in the polarised optical microscopy (POM) image of Figure 2a. Unfortunately, the films did not reveal any field effect behaviour which was ascribed to the detrimental effect of the polar $-\mathrm{OH}$ groups present on the substrate. Subsequently, we proceed to blend the TCNQ with PS of different molecular weights. After an optimisation process, the selected formulation was a $2 \%$ wt. anisole solution of TCNQ:PS280K in a 3:1 weight ratio, which was deposited by BAMS at $105 \stackrel{\circ}{\circ}$ and $10 \mathrm{~mm} / \mathrm{s}$. Crystalline and isotropic films consisting of large plate-like domains were formed (Figure 2b). The devices exhibited an average electron mobility close to $2 \cdot 10^{-3} \mathrm{~cm}^{2} \mathrm{~V}^{-1} \mathrm{~s}$ ${ }^{1}$ with an average threshold voltage of around $10 \mathrm{~V}$. Transfer and output characteristics of the optimised formulation are shown in Figure 2c. Remarkably, the device electrical characteristics were monitored along time and only a decay of $30 \%$ of mobility was observed after one month of fabrication
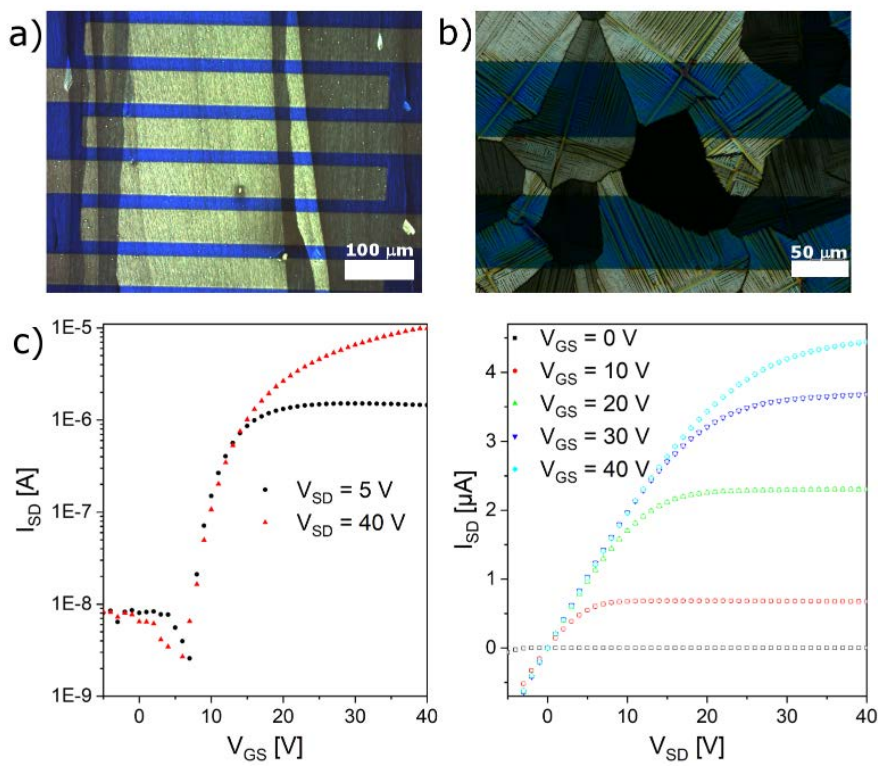

Figure 2. POM images of pure TCNQ (a) and TCNQ:PS280K 3:1 blend (b) films; transfer and output characteristics of TCNQ polymer blend (c).

and storing the devices in environmental conditions (Figure S1). Importantly, this result proves the beneficial effects of using an insulating polymeric matrix for the successful fabrication of TCNQ based n-type OFETs.

The next step was therefore the preparation of thin films of the DBTTF-TCNQ CT. For this purpose, we tested first several solvent mixtures suitable for the growth of DBTTF-TCNQ crystals (Figure S2 and experimental section). For these experiments, it was required to use solvents with a high boiling point, required for BAMS deposition, and also with a certain degree of polarity to avoid the immediate precipitation of the complex after mixing the two components. Two different morphologies were commonly found as shown in the inset of Figure 3a: needle like from chlorobenzene-dimethylformamide (CB-DMF) 50/50 solutions, and plate-like from chlorobenzenebenzonitrile (CB-BZN) 50/50.

Two different polymorphs have previously been described for the CT DBTTF-TCNQ. The a polymorph, prepared by codiffusion of the neutral $D$ and $A$ molecules in an acetonitrile/cyclohexane solvent system, ${ }^{42-44}$ crystallizes as black needle like crystals which belong to a triclinic system 
with donor-acceptor 1:1 ratio. This system arranges in a mixed stack configuration with the direction of charge transfer stack mainly along the $b$ axis and with a shortest interplanar distance of $3.4 \AA$ between the almost planar $\pi$-electron donor and acceptor molecules. Recently, the DBTTF-TCNQ $\beta$ polymorph was also obtained by physical vapour transport. ${ }^{31}$ Unfortunately the $\beta$ crystals, which showed elliptical crystal shape, were too thin to resolve the structure. In order to investigate the crystal phase of the two types of crystals obtained here, we first studied them by XRD, both showing the same known arrangement of the $\alpha$ polymorph (Figure $3 \mathrm{~b}$ ). It is known that the $\beta$ and $\alpha$ polymorphs can be discriminated by Raman spectroscopy. ${ }^{31}$ In fact, the main peak, corresponding to exocyclic $\mathrm{V}_{4} \mathrm{C}=\mathrm{C}$ stretching of the TCNQ moiety, shifts from the $1454 \mathrm{~cm}^{-1}$ value of the neutral acceptor molecule to 1426 $\mathrm{cm}^{-1}$ for the $\alpha$ polymorph and $1448 \mathrm{~cm}^{-1}$ for the $\beta$ structure. All the here-prepared crystals showed the same Raman pattern unambiguously attributable to the $\alpha$ structure as shown in Figure $3 \mathrm{~b}$. The differences in the peaks intensities are only due to different orientations of the $\mathrm{CT}$ complex in the crystal specimens.

DBTTF-TCNQ CT films were prepared on $\mathrm{Si} / \mathrm{SiO}_{2}$ substrates with interdigitated gold electrodes by BAMS and blending its
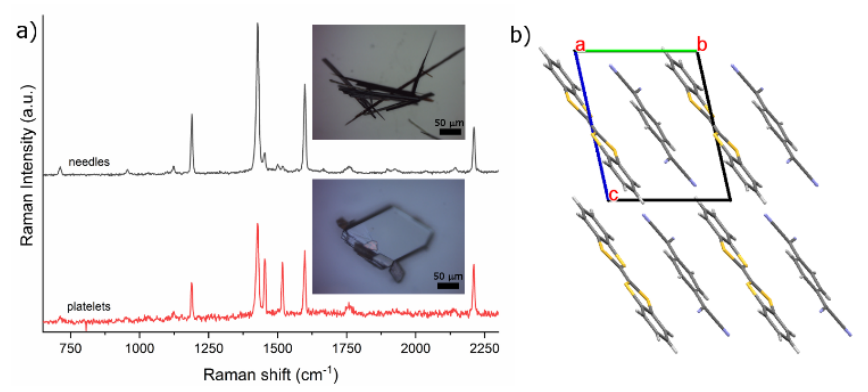

Figure 3. a) Raman spectra of the needle (black line) and plate-like (red line) crystals and morphology of the single crystals. b) Crystal structure of the $\alpha$-DBTTF-TCNQ CT obtained from solution.

components with PS. Different conditions were tested as: DBTTF-TCNQ:PS ratio, PS molecular weight, coating temperature, deposition speed and the solvent and solvents mixtures previously used for the growth of the single crystals (Figure S2). In several conditions homogeneous films with a good degree of crystallinity as observed by POM images (Figure S3) and X-ray diffractograms (Figure S4) were realised. All the devices prepared employing the different conditions were tested as OFETs. The best performances were achieved mixing an equimolar ratio of DBTTF and TCNQ in a solvents mixture CB:BZN 50/50, a DBTTF-TCNQ:PS280k weight ratio of $1: 2$ keeping the concentration of the final solution at $20 \mathrm{~g} / \mathrm{l}$. The deposition was performed at a speed of $1 \mathrm{~mm} / \mathrm{s}$ and keeping the substrate at 150 으. We have previously shown that at this speed the convective crystallization regime dominates and the crystallisation takes place at the meniscus contact line as the solvent is evaporated. ${ }^{45}$ Optical microscope images in bright field and crossed polarized configuration are reported in Figure 4. The POM images of the blended film show a clear orientation of the crystal domains in the direction of the bar shearing. AFM images reported in Figure S5 show that the film morphology is characterized by needles and zones flatter and more homogeneous.

The optimised thin film OFETs exhibited an ambipolar OFET behaviour as shown by the transfer and output curves from Figure 4. The extracted average mobility values were for electrons $\mu_{\mathrm{e}, \mathrm{sat}}=0.6 \cdot 10^{-4} \mathrm{~cm}^{2} \mathrm{~V}^{-1} \mathrm{~s}^{-1}$ and for holes $\mu_{\mathrm{h}, \mathrm{sat}}=2.7 \cdot 10^{-}$ ${ }^{4} \mathrm{~cm}^{2} \mathrm{~V}^{-1} \mathrm{~s}^{-1}$, and the threshold voltages $\left(V_{\mathrm{th}}\right)$ extracted from the respective fits were $-20 \mathrm{~V}$ and $-10 \mathrm{~V}$. Both for electron and hole accumulation transfer curves an off-current quite high of around $100 \mathrm{nA}$ is observed, which is a consequence of a system with intrinsic charges due to the charge transfer between donor and acceptor molecules.

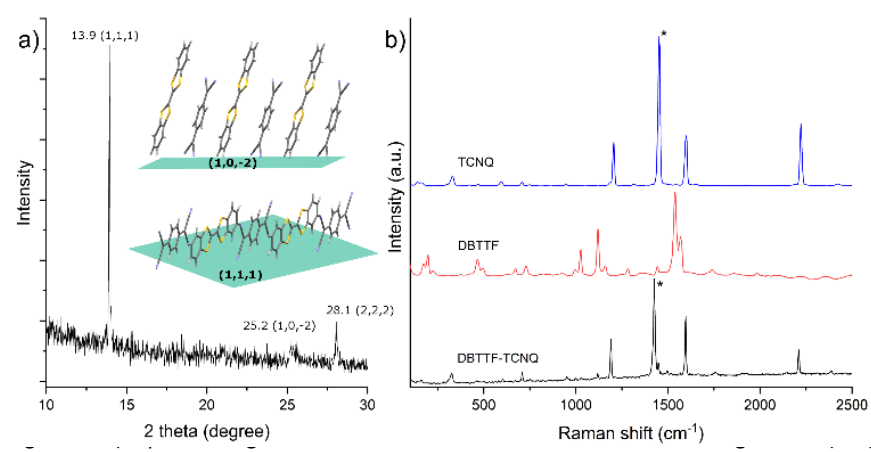

and in cross-polarizer configuration (right); b) Transfer (upper panels) and output (lower panels) characteristics of the optimised formulation of DBTTF-TCNQ:PS280k films.
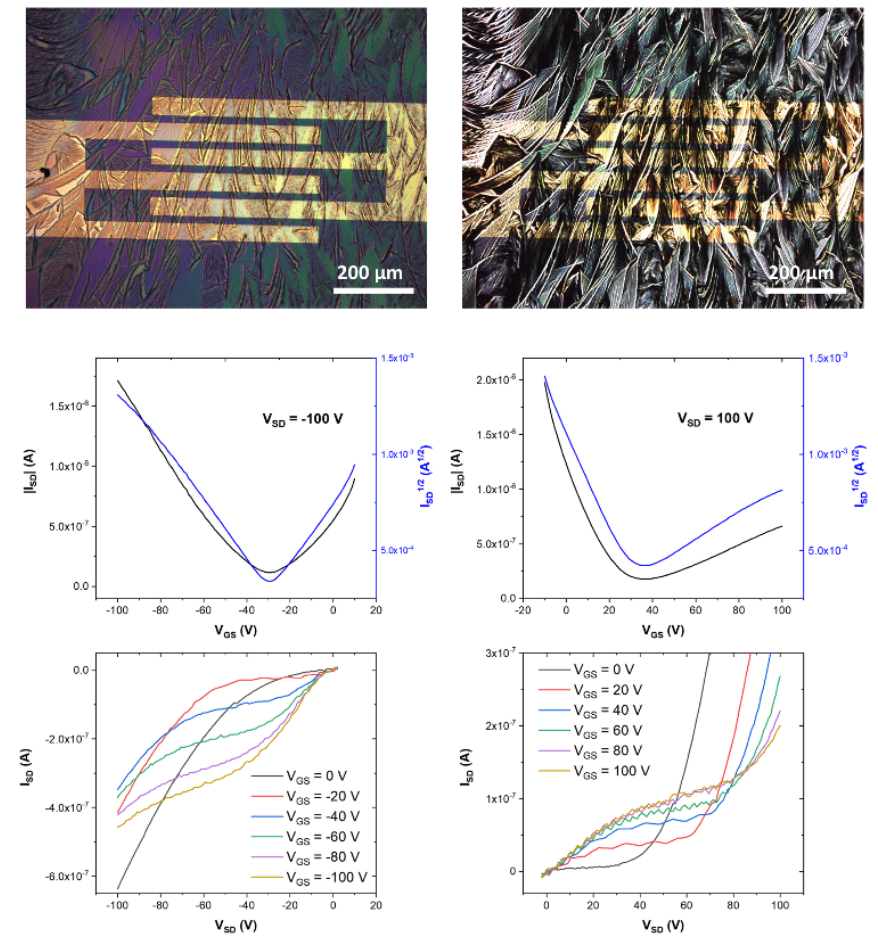

Figure 5. a) XRD of the DBTTF-TCNQ:PS280k film and illustration of the crystal orientations b) Raman spectrum of the DBTTF-TCNQ:PS280k blend film (black line), pure DBTTF (red line) and pure TCNQ (blue line).

For single crystals of both DBTTF-TCNQ polymorphs, an ambipolar transport was reported but with remarkable differences between hole and electron enhancements. ${ }^{31}$ In $\alpha$ DBTTF-TCNQ the electron transport is dominant $\left(\mu_{\mathrm{e}}=0.4 \mathrm{~cm}^{2} \mathrm{~V}\right.$ $\left.{ }^{1} \mathrm{~s}^{-1} ; \mu_{\mathrm{h}}=0.04 \mathrm{~cm}^{2} \mathrm{~V}^{-1} \mathrm{~s}^{-1}\right)$, while the $\beta$-DBTTF-TCNQ polymorph shows a superior hole transport $\left(\mu_{\mathrm{e}}=0.03 \mathrm{~cm}^{2} \mathrm{~V}^{-1} \mathrm{~s}^{-1} ; \mu_{\mathrm{h}}=0.1\right.$ 
$\mathrm{cm}^{2} \mathrm{~V}^{-1} \mathrm{~s}^{-1}$ ). In our films, the mobility found is orders of magnitude lower than the single crystals, which is attributed to the presence of many grain boundaries and crystal defects as well as the fact that the crystals are not completely oriented along the channel (see also below).

To gain in-sights into the thin film polymorphism, the CT films were characterised by XRD and Raman. Figure 5a reports the XRD pattern of the films which were compared with the simulated one from $\alpha$ single-crystal structure. The two main peaks are attributed to the (111) family planes, while the weak peak at 25.2 theta/2theta has been attributed to (01-2) plane, indicating the presence of two specific molecular orientations in the films. XRD data is hence in agreement with a 1:1 stoichiometry between DBTTF and TCNQ and the corresponding d-spacing values from the diffractogram are respectively $6.36 \AA$ (111), $3.53 \AA$ (10-2) and $3.17 \AA$ (222). From these results we can assume that the packing direction of the CT stack is always parallel to the $\mathrm{SiO}_{2}$ dielectric surface, but in different orientations as schematically illustrated in the inset of Figure $5 a$.

To check the crystal phase homogeneity, we performed microRaman investigation of the films with a lateral resolution of 0.5 $\mu \mathrm{m}$. Figure $5 \mathrm{~b}$ displays the Raman spectrum of the CT DBTTFTCNQ:PS280k films along with those of the starting sublimed powders of DBTTF and TCNQ. The Raman spectrum of the CT film presents the characteristic peaks of the parent compounds DBTTF and TCNQ. The main differences are represented by: 1 ) the shift of the charge sensitive vibration modes of the CN group which moves from $2222 \mathrm{~cm}^{-1}$ in TCNQ to $2208 \mathrm{~cm}^{-1}$ in the DBTTF-TCNQ:PS280k film, and 2) the $\mathrm{C}=\mathrm{C}$ vibration that shifts from $1454 \mathrm{~cm}^{-1}$ in TCNQ to $1426 \mathrm{~cm}^{-1}$ in the CT films (marked with an asterisk in Figure 5b). The latter confirms that the DBTTF-TCNQ in the blended film belongs to the $\alpha$ polymorph arrangement, as described above. From the micro-Raman analysis, we were also able to record the single spectra on the needles and on the flat zone areas of the film. As shown in Figure S6, while the characteristic peak of the DBTTF-TCNQ a polymorph remains unaltered at the same position, some other peaks in the range $250-1250 \mathrm{~cm}^{-1}$ show different relative intensities in accordance with the existence of different orientations.

CT complexes are characterized by a partial degree of ionicity. Using spectroscopic techniques, such as infrared and Raman, it is possible to have a valuable estimation of the ionicity of the CT systems under investigation by looking at the shift of the charge sensitive vibrations. ${ }^{15,46-48}$ Regarding the DBTTF-TCNQ system, by Raman spectroscopy it has been reported a degree of ionicity of 0.5 and 0.13 for $\alpha$ and $\beta$ polymorphs, respectively, using the shift of the totally symmetric $\mathrm{a}_{\mathrm{g}} \mathrm{v}_{4}$ mode ( $C=C$ stretching) of the acceptor TCNQ as probe of ionicity. ${ }^{15,46-}$ 48 However, it is well-known that the totally symmetric intramolecular modes couple with the electronic excitation strongly affecting the optical spectra, depending on the crystal arrangement. ${ }^{48-50}$ This effect is known as electron-molecular vibration (e-mv) coupling and, in particular for the specific case of DBTTF-TCNQ, which shows a mixed stack packing arrangement, the Raman-active modes are lowered in frequency by this interaction, giving generally an overestimation of the degree of ionicity. Indeed, in a recent comment, ${ }^{50}$ taking into account such e-mv coupling of the Raman-active molecular vibrations, the degree of ionicity for the system DBTTF-TCNQ has been re-calculated resulting in lower values than the previously estimated ones ${ }^{31}$ and equal to 0.25 and 0.04 for $\alpha$ and $\beta$ polymorphs, respectively.

The best choice for the estimation of the CT degree in DBTTFTCNQ system is the direct observation of the TCNQ $b_{1 u} v_{20} C=C$ stretching mode active in IR which is not influenced by any interaction in regular mixed stack arrangements. In this case it is possible to assume a linear relation between the degree of ionicity and the $\mathrm{C}=\mathrm{C}$ frequency shift. ${ }^{15,47,48}$ In the present work we analysed the films by Infrared Reflection-Absorption Spectroscopy (IRRAS). The C=C IR frequencies of the neutral and total ionized acceptor TCNQ are reported to be respectively 1543 and $1509 \mathrm{~cm}^{-1} .{ }^{15}$ The DBTTF-TCNQ films exhibited the $\mathrm{C}=\mathrm{C}$ shift at $1536 \mathrm{~cm}^{-1}$ in the IR spectra (Figure 6). Therefore, we estimate a degree of ionicity of 0.22 , which is in good agreement with previously mentioned recent estimation for the $\alpha$ structure of DBTTF-TCNQ. ${ }^{50}$ The IR spectrum of the films also show a strong shift of the $\mathrm{CN}$ stretching mode which is also a well-known charge sensitive mode. ${ }^{46}$ However, the vibration frequency of this functional group can be influenced from the chemical interaction environment due to its position at the edge of the molecular moiety and it is known to give a non-reliable or an overestimation of the ionicity. The entire IR spectrum till $7500 \mathrm{~cm}^{-1}$ is reported in Figure $\mathrm{S} 7$ showing the CT band at $5600 \mathrm{~cm}^{-1}(\sim 0.7 \mathrm{eV})$.

It should be noticed that the IRRAS measurements were performed using an incident beam with the electric vector oscillation polarized perpendicular to the sample surface and, hence, only the observation of the out-of-plane modes is possible. The fact that the IRRAS exhibited the $b_{14} \quad C=C$ stretching and the $b_{1 u}$ stretching of the $C N$ group, which is polarized along the long molecular axis of TCNQ, is in agreement with the observation of the peak of the 10-2
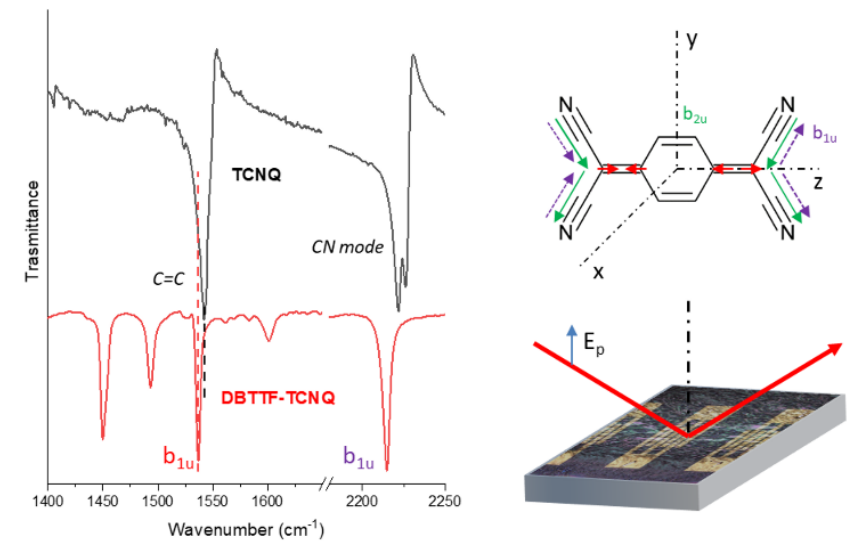

crystallographic plane.

Figure 6. Left: peak positions of the $\mathrm{CN}$ and $\mathrm{C}=\mathrm{C}$ stretching modes in TCNQ (black spectrum) and in DBTTF-TCNQ CT complex (red spectrum). Right: normal modes and symmetries of the analysed vibrational modes of TCNQ moiety (top) and IRRAS experimental configuration (bottom). 


\section{Conclusions}

In conclusion, thin films of the CT complex DBTTF-TCNQ blended with PS were fabricated employing a low cost and scalable solution shearing technique. Structural, spectroscopic and morphological characterization of the devices fabricated confirmed the formation of a homogeneous film with two preferential molecular orientations with respect to the substrate. The devices prepared show almost balanced air stable ambipolar transport with mobility values in the range of $10^{-4} \mathrm{~cm}^{2} \mathrm{~V}^{-1} \mathrm{~s}^{-1}$. Such values are much lower than the previously ones reported for single crystal OFETs, most probably due to the detrimental effect of the two different molecular orientation domains in the film combined with the presence of grain boundaries. Importantly, it was demonstrated the pivotal role of the PS binding polymer which promoted thin film processability and electron transport stability in environmental conditions. Although much efforts are required in order to improve the film quality and device performance, this work opens new perspectives for applications of the long-known CT complexes in the field of OFETs by moving from ideal single crystals to high throughput printed devices.

\section{Conflicts of interest}

There are no conflicts to declare.

\section{Acknowledgements}

This work was funded by European research council (ERC) StG 2012-306826 e-GAMES, Networking Research Center on Bioengineering, Biomaterials, and Nanomedicine (CIBER-BBN), the Spanish Ministry project FANCY CTQ2016-80030-R, the Generalitat de Catalunya (2017SGR918) and the Spanish Ministry of Economy and Competitiveness, through the "Severo Ochoa" Programme for Centers of Excellence in R\&D (SEV-2015-0496). The authors thank Prof. Aldo Brillante and Prof. Alberto Girlando for the fruitful discussions and Dr. Gemma Rius for the access to the Raman Facilities at IMBCNM. T.S. acknowledges the H2020-MSCA-COFUND-2014 Programme (P-SPHERE, Grant agreement 665919).

\section{Notes and references}

1 S. Lee, D. Sasaki, D. Kim, M. Mori, T. Yokota, H. Lee, S. Park, K. Fukuda, M. Sekino, K. Matsuura, T. Shimizu and T. Someya, Nat. Nanotechnol., 2019, 14, 156-160.

2 F. Yang, S. Cheng, X. Zhang, X. Ren, R. Li, H. Dong and W. Hu, Adv. Mater., 2018, 30, 1702415.

3 S. G. Surya, H. N. Raval, R. Ahmad, P. Sonar, K. N. Salama and V. R. Rao, Trends Anal. Chem., 2019, 111, 27-36.

4 E. Menard, V. Podzorov, S.-H. Hur, A. Gaur, M. E. Gershenson and J. A. Rogers, Adv. Mater., 2004, 16, 20972101.
R. Pfattner, S. T. Bromley, C. Rovira and M. Mas-Torrent, Adv. Funct. Mater., 2016, 26, 2256-2275.

Y. Diao, L. Shaw, Z. Bao and S. C. B. Mannsfeld, Energy Environ. Sci., 2014, 7, 2145-2159.

7 M. R. Niazi, R. Li, E. Qiang Li, A. R. Kirmani, M. Abdelsamie, Q. Wang, W. Pan, M. M. Payne, J. E. Anthony, D. M. Smilgies, S. T. Thoroddsen, E. P. Giannelis and A. Amassian, Nat. Commun., 2015, 6, 1-10.

8 S. Riera-Galindo, A. Tamayo and M. Mas-Torrent, ACS Omega, 2018, 3, 2329-2339.

Y. Yuan, G. Giri, A. L. Ayzner, A. P. Zoombelt, S. C. B. Mannsfeld, J. Chen, D. Nordlund, M. F. Toney, J. Huang and Z. Bao, Nat. Commun., 2014, 5, 1-9. M. Kang, H. Hwang, W. T. Park, D. Khim, J. S. Yeo, Y. Kim, Y. J. Kim, Y. Y. Noh and D. Y. Kim, ACS Appl. Mater. Interfaces, 2017, 9, 2686-2692.

R. Pfattner, E. Pavlica, M. Jaggi, S.-X. Liu, S. Decurtins, G. Bratina, J. Veciana, M. Mas-Torrent and C. Rovira, J. Mater. Chem. C, 2013, 1, 3985-3988. S. H. Yu, B. Kang, G. An, B. Kim, M. H. Lee, M. S. Kang, H. Kim, J. H. Lee, S. Lee, K. Cho, J. Y. Lee and J. H. Cho, ACS Appl. Mater. Interfaces, 2015, 7, 2025-2031.

X. Xu, T. Xiao, X. Gu, X. Yang, S. V. Kershaw, N. Zhao, J. Xu and Q. Miao, ACS Appl. Mater. Interfaces, 2015, 7, 2801928026.

T. Leydecker, M. A. Squillaci, F. Liscio, E. Orgiu and P. Samorì, Chem. Mater., DOI:10.1021/acs.chemmater.8b04819.

T. Salzillo, M. Masino, G. Kociok-Köhn, D. Di Nuzzo, E. Venuti, R. G. Della Valle, D. Vanossi, C. Fontanesi, A. Girlando, A. Brillante and E. Da Como, Cryst. Growth Des., 2016, 16, 3028-3036.

P. Hu, K. Du, F. Wei, H. Jiang and C. Kloc, Cryst. Growth Des., 2016, 16, 3019-3027. K. P. Goetz, D. Vermeulen, M. E. Payne, C. Kloc, L. E. McNeil and O. D. Jurchescu, J. Mater. Chem. C, 2014, 2, 30653076.

H. Di Wu, F. X. Wang, Y. Xiao and G. B. Pan, J. Mater. Chem. C, 2013, 1, 2286-2289.

D. Vermeulen, L. Y. Zhu, K. P. Goetz, P. Hu, H. Jiang, C. S. Day, O. D. Jurchescu, V. Coropceanu, C. Kloc and L. E. Mcneil, J. Phys. Chem. C, 2014, 118, 24688-24696.

A. Mandal, P. Swain, B. Nath, S. Sau and P. Mal, CrystEngComm, 2019, 21, 981-989. Z. Shuai and D. Zhu, Adv. Mater., 2014, 26, 4093-4099. J. Zhang, J. Tan, Z. Ma, W. Xu, G. Zhao, H. Geng, C. Di, W. $\mathrm{Hu}$, Z. Shuai, K. Singh and D. Zhu, J. Am. Chem. Soc., 2013, 135, 558-561.

L. Zhu, Y. Yi, Y. Li, E. G. Kim, V. Coropceanu and J. L. Brédas, J. Am. Chem. Soc., 2012, 134, 2340-2347.

J. Zhang, J. Jin, H. Xu, Q. Zhang and W. Huang, J. Mater. Chem. C, 2018, 6, 3485-3498. R. Sato, M. Dogishi, T. Higashino, T. Kadoya, T. Kawamoto and T. Mori, J. Phys. Chem. C, 2017, 121, 6561-6568. J. Zhang, W. Xu, P. Sheng, G. Zhao and D. Zhu, Acc. Chem. Res., 2017, 50, 1654-1662. Y. Shibata, J. Tsutsumi, S. Matsuoka, K. Matsubara, Y. 
Yoshida, M. Chikamatsu and T. Hasegawa, Appl. Phys. Lett., 2015, 106, 0-4. H. Jiang, P. Hu, J. Ye, K. K. Zhang, Y. Long, W. Hu and C. Kloc, J. Mater. Chem. C, 2018, 6, 1884-1902.

L. Sun, W. Zhu, F. Yang, B. Li, X. Ren, X. Zhang and W. Hu, Phys. Chem. Chem. Phys., 2018, 20, 6009-6023. Y. Wang, W. Zhu, H. Dong, X. Zhang, R. Li and W. Hu, Top. Curr. Chem., , DOI:10.1007/s41061-016-0081-8. K. P. Goetz, J. Tsutsumi, S. Pookpanratana, J. Chen, N. S. Corbin, R. K. Behera, V. Coropceanu, C. A. Richter, C. A. Hacker, T. Hasegawa and O. D. Jurchescu, Adv. Electron. Mater., 2016, 2, 1-10. K. Shibata, K. Ishikawa, H. Takezoe, H. Wada and T. Mori, Appl. Phys. Lett., 2008, 92, 023305. Y. Takahashi, T. Hasegawa, Y. Abe, Y. Tokura, K. Nishimura and G. Saito, Appl. Phys. Lett., 2005, 86, 063504. Y. Takahashi, T. Hasegawa, Y. Abe, Y. Tokura and G. Saito, Appl. Phys. Lett., 2006, 88, 073504.

F. G. del Pozo, S. Fabiano, R. Pfattner, S. Georgakopoulos, S. Galindo, X. Liu, S. Braun, M. Fahlman, J. Veciana, C. Rovira, X. Crispin, M. Berggren and M. Mas-Torrent, Adv. Funct. Mater., 2016, 26, 2379-2386. I. Temiño, F. G. Del Pozo, M. R. Ajayakumar, S. Galingo, J. Puigdollers and M. Mas-Torrent, Adv. Mater. Technol., 2016, 1, 1600090. S. Riera-Galindo, F. Leonardi, R. Pfattner and M. MasTorrent, Adv. Mater. Technol., 2019, 1900104. Zhao, O. Wodo, D. Ren, H. U. Khan, M. R. Niazi, H. Hu, M. Abdelsamie, R. Li, E. Q. Li, L. Yu, B. Yan, M. M. Payne, J. Smith, J. E. Anthony, T. D. Anthopoulos, S. T. Thoroddsen, B. Ganapathysubramanian and A. Amassian, Adv. Funct. Mater., 2016, 26, 1737-1746. A. Campos, S. Riera-Galindo, J. Puigdollers and M. MasTorrent, ACS Appl. Mater. Interfaces, 2018, 10, 1595215961. F. Leonardi, S. Casalini, Q. Zhang, S. Galindo, D. Gutiérrez and M. Mas-Torrent, Adv. Mater., 2016, 28, 10311-10316. M. Yamagishi, Y. Tominari, T. Uemura and J. Takeya, Appl. Phys. Lett., 2009, 94, 1-4. H. Kobayashi and J. Nakayama, Bull. Chem. Soc. Jpn., 1981, 54, 2408-2411. T. J. Kistenmacher, T. J. Emge, F. M. Wiygul, W. A. Bryden, J. S. Chappell, J. P. Stokes, L.-Y. Chiang and D. O. Cowan, Solid State Commun., 1981, 39, 415-417. . Bloch, J. P. Ferraris, D. O. Cowan and T. J. Kistenmacher, Mol. Cryst. Liq. Cryst., 1982, 87, 137-161. S. Galindo, A. Tamayo, F. Leonardi and M. Mas-Torrent, Adv. Funct. Mater., 2017, 27, 1-9.

Nanova, S. Beck, A. Fuchs, T. Glaser, C. Lennartz, W. Kowalsky, A. Pucci and M. Kroeger, Org. Electron. physics, Mater. Appl., 2012, 13, 1237-1244. J. Henderson, M. Masino, L. E. Hatcher, G. Kociok-Köhn, T. Salzillo, A. Brillante, P. R. Raithby, A. Girlando and E. Da Como, Cryst. Growth Des., 2018, 18, 2003-2009.

T. Salzillo, R. G. Della Valle, E. Venuti, G. Kociok-Köhn, M.
$516,45-50$.

A. Girlando, A. Painelli and C. Pecile, Mol. Cryst. Liq. Cryst., 1985, 120, 17-26.

A. Girlando, Adv. Electron. Mater., 2017, 3, 1600437. Masino, A. Girlando and A. Brillante, J. Cryst. Growth, 2019, 